\title{
The prevalence and correlates of taking folic acid and vitamin supplements among adults aged $\geq 45$ years with CVD
}

\author{
Guixiang Zhao ${ }^{1, *}$, Earl S Ford ${ }^{1}$, Chaoyang Li ${ }^{1}$ and Ali H Mokdad ${ }^{2}$ \\ 'Division of Adult and Community Health, National Center for Chronic Disease Prevention and Health \\ Promotion, Centers for Disease Control and Prevention, 4770 Buford Highway, Mailstop K66, Atlanta, \\ GA 30341, USA: ${ }^{2}$ Institute for Health Metrics and Evaluation, University of Washington, Seattle, \\ WA, USA
}

Submitted 2 November 2009: Accepted 20 January 2010: First published online 3 March 2010

\begin{abstract}
Objective: To examine the prevalence and likelihood of taking folic acid or vitamin supplements among adults with CHD or stroke $v$. adults without these conditions.

Design: A cross-sectional Behavioural Risk Factor Surveillance System survey was conducted in twelve states of the United States and Puerto Rico in 2006. Selfreported data from participants were collected.

Setting: The United States.

Subjects: US non-institutionalised adults ( $n$ 41792), aged $\geq 45$ years.

Results: Of all participants, 5445 had CHD and 2076 had stroke. Significantly higher percentages of women than men reported taking folic acid or vitamin supplements. After adjustment for potential confounders, women with CHD had a significantly lower adjusted prevalence (AP) and adjusted OR (AOR) than women without CHD for taking folic acid less than one time per $\mathrm{d}(\mathrm{AP}=3.9 \% v .5 \cdot 5 \% ; \mathrm{AOR}=0 \cdot 56 ; 95 \%$ CI $0 \cdot 39,0 \cdot 81$ ), for taking folic acid one to four times per $\mathrm{d}(\mathrm{AP}=50 \cdot 0 \% v .57 \cdot 5 \%$; $\mathrm{AOR}=0 \cdot 68 ; 95 \% \mathrm{CI} 0 \cdot 60,0 \cdot 79)$, and for taking vitamin supplements (AP $=60 \cdot 9 \%$ v. $69 \cdot 9 \%$; AOR $=0 \cdot 66 ; 95 \%$ CI 0.57, 0.76). Men with CHD had a significantly higher $\mathrm{AP}(50 \cdot 4 \% v \cdot 46 \cdot 2 \%)$ and $\mathrm{AOR}(1 \cdot 17 ; 95 \% \mathrm{CI} 1 \cdot 02,1 \cdot 33)$ of taking folic acid one to four times per $d$ than men without CHD. In both sexes, adults with stroke were as likely as those without to take these supplements.

Conclusions: Substantial variations in the prevalence and likelihood of taking folic acid or vitamin supplements exist by gender and by CHD status, but not by stroke status.
\end{abstract}

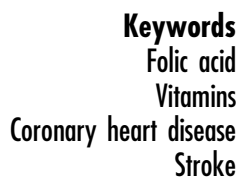

Folic acid

Vitamins

Stroke
Although the mortality rate of patients with CHD has decreased by $52 \%$ in men and $49 \%$ in women from 1980 to $2002^{(1)}$ and the mortality rate of stroke decreased by $24 \cdot 2 \%$ from 1994 to $2004^{(2)}$, the burden of these diseases remains high. Among US adults, the prevalence of CHD was $7 \cdot 3 \%$ and the prevalence of stroke was $2 \cdot 6 \%$ in 2005.

Hyperhomocysteinaemia is associated with an increased risk for $\mathrm{CVD}^{(3-5)}$. Results from meta-analyses have shown that for a $5 \mu \mathrm{mol} / 1$ increase in serum homocysteine levels, the risk of myocardial infarction increased by $84 \%{ }^{(5)}$, the risk of $\mathrm{CHD}$ increased by $23-70 \%{ }^{(4)}$, and the risk of cerebrovascular disease increased by $58-116 \%{ }^{(4)}$, although prospective studies offer weaker support for this association than case-control studies ${ }^{(4)}$. A higher intake of folic acid or multivitamins reduces serum homocysteine levels $^{(6,7)}$ and is associated with reduced risk of acute coronary events and myocardial infarction ${ }^{(8-11)}$ or reduced rates of coronary restenosis and revascularisation in patients with coronary angioplasty ${ }^{(12,13)}$. However, other studies including recent clinical trials failed to show any beneficial effects on the risk of recurrent CVD or on total CVD or CHD mortality when using folic acid and $B$ vitamins to lower homocyteine levels among patients with pre-existing CVD or renal disease ${ }^{(14-17)}$ or among people at high risk for CVD (having a history of vascular disease, diabetes or other coronary risk factors ${ }^{(18,19)}$.

From 1990-1997 to 1998-2002, stroke-related mortality has decreased dramatically in the United States and Canada ${ }^{(20)}$. Folic acid fortification of flour and cereal grain initiated in 1998 may have contributed to this decline ${ }^{(21)}$. In addition, folic acid supplementation could effectively reduce the risk of stroke in primary prevention ${ }^{(22-25)}$; 
however, its role in the secondary prevention of stroke remains to be elucidated.

The National Health and Nutrition Examination Survey (NHANES) data have shown that dietary supplement use increased from $23 \%$ in NHANES I (1971-1975) to $40 \%$ in NHANES III $(1988-1994)^{(26)}$. The 1999-2000 NHANES data also showed that $52 \%$ of US adults reported taking dietary supplements in the past month, and $35 \%$ reported regular use of multivitamin-multimineral supplements ${ }^{(26)}$. However, the prevalence and correlates of taking folic acid and vitamin supplements among people with CVD are not well understood. Using a relatively large survey sample, we examined the percentage and likelihood of adults with CHD or stroke who reported taking folic acid or vitamin supplements in comparison with those observed in adults without these conditions.

\section{Methods}

Data for our analyses came from the 2006 Behavioural Risk Factor Surveillance System (BRFSS), a population-based telephone survey of health-related behaviours regarding the leading causes of death among non-institutionalised US adults aged $\geq 18$ years. The survey was reviewed by the Human Research Protection Office at the Centers for Disease Control and Prevention and was determined to be exempt from human subject guidelines. The BRFSS survey design, sampling methods and weights have been described elsewhere ${ }^{(27)}$, and BRFSS data have consistently been found to provide valid and reliable estimates when compared with other national household surveys in the United States ${ }^{(27-29)}$. Further information on BRFSS is available at http://www.cdc.gov/brfss/.

We analysed the data collected from survey participants aged $\geq 45$ years in twelve states (Delaware, Florida, Georgia, Kentucky, Minnesota, Missouri, Montana, New York, North Carolina, Virginia, Wisconsin and Wyoming) and Puerto Rico that had an optional module on folic acid and vitamin supplementation in the 2006 BRFSS. The median cooperation rate (the percentage of eligible persons contacted who completed the interview) for the 2006 BRFSS was $74.5 \%$.

Vitamin supplementation was assessed by asking participants whether they were currently taking any vitamin pills or supplements. Folic acid supplementation was assessed by asking participants whether and how often they were taking multivitamins or any vitamin pills/ supplements that contained folic acid. Their responses were then categorised as (i) no folic acid supplements, (ii) taking folic acid supplements less than one time per $\mathrm{d}$ and (iii) taking folic acid supplements one to four times per $\mathrm{d}$. Those who answered that they were taking folic acid supplements more than four times per $d$ were excluded from the analyses because there were few participants in this category $(<0.5 \%)$. Self-reported supplement use of vitamins and minerals has been highly reliable and valid ${ }^{(30)}$.

Participants' CVD status was assessed by asking them whether they had ever been told by a doctor or other health professional that they had CHD (including heart attack or myocardial infarction, or angina pectoris) or stroke. Self-reports of physician-diagnosed CHD and stroke have an adequate validity and reliability compared with those obtained through medical records ${ }^{(31-32)}$.

The demographic variables in our analyses included age, sex, BMI $\left(\mathrm{kg} / \mathrm{m}^{2}\right.$; self-reported), race/ethnicity (nonHispanic white, non-Hispanic black, Hispanic and others), education (below high school diploma, high school graduate, some college or technical school and above college graduate) and household income $(<\$ 25000$, $\$ 25000$ to $<\$ 50000$ and $\geq \$ 50000$ ). In addition, participants' status concerning smoking and alcohol consumption was included as a covariate in our analyses. For smoking status, participants were categorised as (i) current smokers (participants who had smoked 100 or more cigarettes during their lifetime and were still smoking), (ii) former smokers (participants who had smoked 100 or more cigarettes in their entire life but stopped) and (iii) never smoking (participants who had smoked less than 100 cigarettes during their lifetime). Heavy drinkers were defined as women who had one or more drinks per $\mathrm{d}$ or men who had two or more drinks per $\mathrm{d}$.

\section{Statistical analyses}

After excluding from analyses the participants who responded 'don't know/not sure', refused to answer or had missing responses for any of the study variables, a total of 41792 participants remained in our analyses. The prevalence estimates for taking folic acid or vitamin supplements by CHD or stroke were age-adjusted to the standard 2000 US population. Logistic regression analyses were conducted to estimate the adjusted prevalence (AP; predicted marginal probability) of taking folic acid or vitamin supplements and to estimate the adjusted OR (AOR) with $95 \% \mathrm{CI}$ to assess associations of taking folic acid or vitamin supplements with CVD after adjustment for covariates. We used the Survey Data Analysis (SUDAAN) statistical software package release $9 \cdot 0$ (Research Triangle Institute, Research Triangle Park, NC, USA) to account for the multistage, disproportionate stratified sampling design.

\section{Results}

Of all participants, a total of 5445 (12.3\% (weighted), 2756 men $(15 \cdot 2 \%)$ and 2689 women $(9 \cdot 6 \%)$ ) had CHD and $2076(4 \cdot 4 \%$ (weighted), 867 men $(4 \cdot 2 \%)$ and 1209 women $(4.5 \%))$ had stroke. The characteristics of participants with or without these conditions varied significantly by sociodemographics (Table 1). Overall, those 
Table 1 Sociodemographic characteristics of study population by CHD and stroke, BRFSS, 2006

\begin{tabular}{|c|c|c|c|c|c|c|c|c|c|c|}
\hline & \multicolumn{5}{|c|}{$\mathrm{CHD}$} & \multicolumn{5}{|c|}{ Stroke } \\
\hline & \multicolumn{2}{|c|}{ Yes (n 5445) } & \multicolumn{2}{|c|}{ No $(n 36347)$} & \multirow[b]{2}{*}{$P$ value* } & \multicolumn{2}{|c|}{ Yes (n 2076) } & \multicolumn{2}{|c|}{ No $(n 39716)$} & \multirow[b]{2}{*}{$P$ value* } \\
\hline & $\%$ & SE & $\%$ & SE & & $\%$ & SE & $\%$ & SE & \\
\hline Age (years) & & & & & $<0.001$ & & & & & $<0.001$ \\
\hline 45 to $<55$ & $16 \cdot 5$ & $0 \cdot 8$ & $42 \cdot 9$ & $0 \cdot 4$ & & $17 \cdot 4$ & 1.5 & $40 \cdot 7$ & $0 \cdot 4$ & \\
\hline 55 to $<65$ & $27 \cdot 7$ & $1 \cdot 0$ & $29 \cdot 0$ & $0 \cdot 4$ & & $25 \cdot 7$ & $1 \cdot 6$ & $29 \cdot 0$ & $0 \cdot 4$ & \\
\hline$\geq 65$ & $55 \cdot 8$ & $1 \cdot 1$ & $28 \cdot 1$ & 0.3 & & $56 \cdot 9$ & $1 \cdot 8$ & $30 \cdot 3$ & $0 \cdot 3$ & \\
\hline Sex & & & & & $<0.001$ & & & & & $0 \cdot 238$ \\
\hline Men & $60 \cdot 6$ & $1 \cdot 0$ & $47 \cdot 6$ & $0 \cdot 4$ & & $51 \cdot 3$ & $1 \cdot 8$ & $49 \cdot 1$ & $0 \cdot 4$ & \\
\hline Women & $39 \cdot 4$ & $1 \cdot 0$ & $52 \cdot 4$ & $0 \cdot 4$ & & $48 \cdot 7$ & $1 \cdot 8$ & $50 \cdot 9$ & $0 \cdot 4$ & \\
\hline Race/ethnicity & & & & & $0 \cdot 129$ & & & & & 0.023 \\
\hline Non-Hispanic white & $76 \cdot 1$ & $1 \cdot 0$ & $76 \cdot 1$ & $0 \cdot 4$ & & $74 \cdot 2$ & $1 \cdot 8$ & $76 \cdot 2$ & 0.4 & \\
\hline Non-Hispanic black & $8 \cdot 2$ & $0 \cdot 6$ & $9 \cdot 4$ & $0 \cdot 3$ & & $11 \cdot 8$ & $1 \cdot 3$ & $9 \cdot 2$ & $0 \cdot 3$ & \\
\hline Hispanic & $9 \cdot 9$ & $0 \cdot 6$ & $9 \cdot 9$ & 0.3 & & $7 \cdot 5$ & $1 \cdot 2$ & $10 \cdot 0$ & $0 \cdot 2$ & \\
\hline Others & $5 \cdot 8$ & $0 \cdot 6$ & $4 \cdot 6$ & $0 \cdot 2$ & & $6 \cdot 5$ & $1 \cdot 1$ & $4 \cdot 6$ & $0 \cdot 2$ & \\
\hline Education & & & & & $<0.001$ & & & & & $<0.001$ \\
\hline$<$ High school diploma & $18 \cdot 5$ & $0 \cdot 8$ & $9 \cdot 7$ & 0.2 & & $22 \cdot 3$ & $1 \cdot 6$ & $10 \cdot 3$ & $0 \cdot 2$ & \\
\hline High school graduate & $32 \cdot 5$ & $1 \cdot 0$ & $29 \cdot 0$ & $0 \cdot 4$ & & $34 \cdot 8$ & $1 \cdot 7$ & $29 \cdot 2$ & $0 \cdot 3$ & \\
\hline Some college/technical & $24 \cdot 4$ & 0.9 & $25 \cdot 1$ & $0 \cdot 4$ & & $21 \cdot 8$ & $1 \cdot 4$ & $25 \cdot 2$ & $0 \cdot 3$ & \\
\hline$\geq$ College graduate & $24 \cdot 6$ & $1 \cdot 0$ & $36 \cdot 2$ & $0 \cdot 4$ & & $21 \cdot 1$ & $1 \cdot 6$ & $35 \cdot 3$ & 0.4 & \\
\hline $\operatorname{BMl}\left(\mathrm{kg} / \mathrm{m}^{2}\right)$ & & & & & $<0.001$ & & & & & 0.008 \\
\hline$<25$ & $27 \cdot 1$ & $1 \cdot 0$ & $32 \cdot 9$ & $0 \cdot 4$ & & $33 \cdot 1$ & $1 \cdot 8$ & $32 \cdot 2$ & $0 \cdot 4$ & \\
\hline 25 to $<30$ & $39 \cdot 6$ & $1 \cdot 1$ & $40 \cdot 6$ & 0.4 & & $35 \cdot 7$ & $1 \cdot 7$ & $40 \cdot 7$ & 0.4 & \\
\hline$\geq 30$ & $33 \cdot 3$ & $1 \cdot 0$ & $26 \cdot 5$ & $0 \cdot 4$ & & $31 \cdot 3$ & $1 \cdot 6$ & $27 \cdot 2$ & $0 \cdot 3$ & \\
\hline Income (\$) & & & & & $<0.001$ & & & & & $<0.001$ \\
\hline$<25000$ & $42 \cdot 8$ & $1 \cdot 0$ & $25 \cdot 1$ & 0.3 & & $53 \cdot 7$ & $1 \cdot 8$ & $26 \cdot 0$ & $0 \cdot 3$ & \\
\hline 25000 to $<50000$ & $30 \cdot 4$ & $1 \cdot 0$ & $28 \cdot 2$ & $0 \cdot 4$ & & $26 \cdot 9$ & $1 \cdot 6$ & $28 \cdot 6$ & $0 \cdot 3$ & \\
\hline$\geq 50000$ & $26 \cdot 8$ & $1 \cdot 0$ & $46 \cdot \overline{7}$ & $0 \cdot 4$ & & $19 \cdot 4$ & $1 \cdot 5$ & $45 \cdot 4$ & 0.4 & \\
\hline
\end{tabular}

BRFSS, Behavioral Risk Factor Surveillance System.

${ }^{*} \chi^{2}$ tests.

who reported having CHD or stroke were more likely to be older and to have a lower household income. Significantly more men than women reported having CHD $(P<0 \cdot 001)$, but not stroke $(P=0 \cdot 238)$. In addition, the percentages of adults with CHD or stroke differed significantly by participants' educational and BMI levels. A significant racial/ethnic difference existed among participants who reported having stroke (Table $1, P<0.05$ ).

Overall, $3.9 \%$ of men and $5.0 \%$ of women reported taking folic acid supplements less than one time per $\mathrm{d}$, $49 \cdot 2 \%$ of men and $58.4 \%$ of women reported taking folic acid supplements one to four times per $\mathrm{d}$ and $58.8 \%$ of men and $70 \cdot 2 \%$ of women reported taking vitamin supplements (Table 2). By states (and US territories), the prevalence of taking folic acid supplements ranged from $1.5 \%$ in Kentucky to $6.9 \%$ in Puerto Rico for les than one time per $\mathrm{d}$ and from $38.2 \%$ in Puerto Rico to $60.3 \%$ in Montana for one to four times per $\mathrm{d}$; the prevalence of taking vitamin supplements ranged from $52.7 \%$ in Puerto Rico to $73.0 \%$ in Montana.

Women with CHD had a significantly lower prevalence (age-adjusted) of taking folic acid supplements or taking vitamin supplements than women without $\operatorname{CHD}(P<0 \cdot 01$ for all comparisons). Among both men and women, the prevalence of taking folic acid supplements one to four times per $\mathrm{d}$ or taking vitamin supplements was significantly lower in those with stroke than in those without $(P<0.05$ for both). In addition, in both men and women, regardless of their disease status, the percentages of adults who were taking folic acid supplements one to four times per $\mathrm{d}$ or taking vitamin supplements increased significantly with increases in age, educational levels and household income, but decreased when BMI levels increased. NonHispanic whites had the highest and current smokers had the lowest prevalence of taking folic acid supplements one to four times per $\mathrm{d}$ or taking vitamin supplements among the respective characteristics. Women who were heavy drinkers had a significantly higher prevalence of taking folic acid (one to four times per d) or taking vitamin supplements than women who were not heavy drinkers (Table 2).

Multivariate analyses showed a significant gender interaction on the association of taking folic acid or vitamin supplements with $\mathrm{CHD}(P<0 \cdot 01)$, but not with stroke. After adjustment for age, race/ethnicity, education, BMI, household income, smoking and heavy alcohol consumption, the AP and the AOR for taking folic acid supplements one to four times per d among men with CHD were significantly higher than among those without $(P<0.05$ for both, Table 3$)$. In contrast, the AP and the AOR for taking folic acid supplements among women with CHD were significantly lower than among those without $(P<0 \cdot 001$ for both, Table 3). In addition, women with CHD were significantly less likely to take vitamin supplements than those without CHD $(P<0 \cdot 001$, Table 4). Adults with stroke were as likely to take folic acid or 
Table 2 Age-adjusted prevalence of taking any vitamins or folic acid-containing vitamins among US men and women aged $\geq 45$ years, by selected characteristics, BRFSS, 2006

\begin{tabular}{|c|c|c|c|c|c|c|c|c|c|c|c|c|}
\hline & \multicolumn{6}{|c|}{ Men } & \multicolumn{6}{|c|}{ Women } \\
\hline & \multicolumn{4}{|c|}{ Folic acid-containing vitamins (times/d) } & & & \multicolumn{4}{|c|}{ Folic acid-containing vitamins (times/d) } & & \\
\hline & \multicolumn{2}{|c|}{$>0$ to $<1$} & \multicolumn{2}{|c|}{$1-4$} & \multicolumn{2}{|c|}{ Any vitamins } & \multicolumn{2}{|c|}{$>0$ to $<1$} & \multicolumn{2}{|c|}{$1-4$} & \multicolumn{2}{|c|}{ Any vitamins } \\
\hline & $\%$ & SE & $\%$ & SE & $\%$ & SE & $\%$ & SE & $\%$ & SE & $\%$ & SE \\
\hline $\begin{array}{l}\text { Total } \\
\text { CHD* }^{*}\end{array}$ & $3 \cdot 9$ & $0 \cdot 2$ & $49 \cdot 2$ & $0 \cdot 6$ & $58 \cdot 8$ & $0 \cdot 6$ & $5 \cdot 0$ & $0 \cdot 2$ & $58 \cdot 4$ & $0 \cdot 5$ & $70 \cdot 2$ & 0.5 \\
\hline Yes & $3 \cdot 0 t$ & $0 \cdot 6$ & $51 \cdot 8$ & $1 \cdot 5$ & $58 \cdot 6$ & $1 \cdot 5$ & $3 \cdot 3 \ddagger$ & $0 \cdot 7$ & $47 \cdot 6 \S$ & $1 \cdot 7$ & $57 \cdot 6 \S$ & $1 \cdot 7$ \\
\hline No & $4 \cdot 0$ & $0 \cdot 3$ & $48 \cdot 7$ & $0 \cdot 7$ & $58 \cdot 7$ & $0 \cdot 7$ & $5 \cdot 1$ & $0 \cdot 2$ & $59 \cdot 7$ & $0 \cdot 6$ & $71 \cdot 6$ & 0.5 \\
\hline \multicolumn{13}{|l|}{ Stroke* } \\
\hline Yes & $3 \cdot 5$ & $1 \cdot 4$ & $43 \cdot 2 \|$ & $3 \cdot 0$ & $52 \cdot 7 \|$ & $3 \cdot 1$ & $4 \cdot 4$ & $0 \cdot 9$ & $53 \cdot 2 \|$ & $2 \cdot 4$ & $63 \cdot 6 \ddagger$ & $2 \cdot 4$ \\
\hline No & $3 \cdot 9$ & $0 \cdot 2$ & $49 \cdot 5$ & $0 \cdot 6$ & $59 \cdot 1$ & $0 \cdot 6$ & $5 \cdot 0$ & $0 \cdot 2$ & $58 \cdot 6$ & 0.5 & $70 \cdot 6$ & 0.5 \\
\hline \multicolumn{13}{|l|}{ Age (years) } \\
\hline 45 to $<55$ & $7 \cdot 2$ & $0 \cdot 6$ & $39 \cdot 5$ & $1 \cdot 0$ & $50 \cdot 6$ & $1 \cdot 0$ & $7 \cdot 1$ & $0 \cdot 4$ & $50 \cdot 3$ & $0 \cdot 8$ & $63 \cdot 5$ & $0 \cdot 8$ \\
\hline 55 to $<65$ & $3 \cdot 7$ & $0 \cdot 4$ & $50 \cdot 2$ & $1 \cdot 1$ & $59 \cdot 0$ & $1 \cdot 1$ & $5 \cdot 4$ & $0 \cdot 4$ & $58 \cdot 5$ & 0.9 & $70 \cdot 5$ & 0.8 \\
\hline$\geq 65$ & $2 \cdot 2$ & $0 \cdot 3$ & $53 \cdot 6$ & $1 \cdot 0$ & $63 \cdot 3$ & $1 \cdot 0$ & $3 \cdot 4$ & 0.3 & $62 \cdot 9$ & $0 \cdot 8$ & $73 \cdot 7$ & $0 \cdot 8$ \\
\hline$P$ value for a trend & \multicolumn{2}{|c|}{$<0.001$} & \multicolumn{2}{|c|}{$<0.001$} & \multicolumn{2}{|c|}{$<0.001$} & \multicolumn{2}{|c|}{$<0.001$} & \multicolumn{2}{|c|}{$<0.001$} & \multicolumn{2}{|c|}{$<0.001$} \\
\hline Race/ethnicity** & & & & & & & & & & & & \\
\hline Non-Hispanic white & $3 \cdot 6$ & $0 \cdot 2$ & $52 \cdot 3 \|$ & $0 \cdot 7$ & $61 \cdot 7 \|$ & $0 \cdot 6$ & $4 \cdot 7$ & $0 \cdot 2$ & $61 \cdot 8 \ddagger$ & $0 \cdot 5$ & $73 \cdot 1 \ddagger$ & 0.5 \\
\hline Non-Hispanic black & $4 \cdot 8$ & $1 \cdot 3$ & $36 \cdot 7$ & $2 \cdot 6$ & $47 \cdot 4$ & $2 \cdot 7$ & $6 \cdot 4$ & 0.9 & $46 \cdot 5$ & $2 \cdot 0$ & $58 \cdot 0$ & $2 \cdot 0$ \\
\hline Hispanic & $4 \cdot 3$ & $0 \cdot 9$ & $37 \cdot 4$ & $2 \cdot 2$ & $47 \cdot 5$ & $2 \cdot 3$ & $5 \cdot 8$ & $0 \cdot 7$ & $46 \cdot 4$ & $1 \cdot 9$ & $61 \cdot 2$ & 1.9 \\
\hline Others & $5 \cdot 5$ & $1 \cdot 6$ & $43 \cdot 6$ & $3 \cdot 7$ & $52 \cdot 9$ & $3 \cdot 7$ & $5 \cdot 0$ & 1.5 & $50 \cdot 5$ & $3 \cdot 6$ & $64 \cdot 0$ & $3 \cdot 3$ \\
\hline \multicolumn{13}{|l|}{ Education } \\
\hline$<$ High school diploma & $3 \cdot 0$ & $0 \cdot 8$ & $30 \cdot 7$ & $1 \cdot 7$ & $41 \cdot 2$ & $1 \cdot 9$ & $4 \cdot 9$ & $1 \cdot 0$ & $40 \cdot 9$ & $1 \cdot 6$ & $52 \cdot 4$ & $1 \cdot 6$ \\
\hline High school graduate & $3 \cdot 2$ & 0.5 & $44 \cdot 3$ & $1 \cdot 1$ & $53 \cdot 0$ & $1 \cdot 2$ & $3 \cdot 3$ & $0 \cdot 3$ & $55 \cdot 5$ & 0.9 & $65 \cdot 2$ & 0.9 \\
\hline Some college/technical & $3 \cdot 9$ & $0 \cdot 4$ & $49 \cdot 6$ & $1 \cdot 3$ & $59 \cdot 8$ & $1 \cdot 3$ & $5 \cdot 6$ & $0 \cdot 5$ & $62 \cdot 6$ & $1 \cdot 0$ & $75 \cdot 2$ & $0 \cdot 8$ \\
\hline$\geq$ College graduate & $4 \cdot 5$ & 0.4 & $57 \cdot 7$ & $1 \cdot 0$ & $67 \cdot 1$ & $1 \cdot 0$ & $6 \cdot 3$ & 0.5 & $64 \cdot 3$ & $1 \cdot 0$ & $77 \cdot 6$ & 0.9 \\
\hline$P$ value for a trend & \multirow{2}{*}{\multicolumn{2}{|c|}{0.059}} & \multicolumn{2}{|c|}{$<0.001$} & $<0 \cdot c$ & & & & & & $<0.0$ & 01 \\
\hline BMI $\left(\mathrm{kg} / \mathrm{m}^{2}\right)$ & & & & & & & & & & & & \\
\hline$<25$ & $5 \cdot 2$ & $0 \cdot 7$ & $51 \cdot 0$ & $1 \cdot 3$ & $62 \cdot 0$ & $1 \cdot 2$ & $5 \cdot 4$ & $0 \cdot 4$ & $62 \cdot 1$ & 0.9 & $74 \cdot 5$ & $0 \cdot 8$ \\
\hline 25 to $<30$ & $3 \cdot 4$ & $0 \cdot 3$ & $50 \cdot 4$ & 0.9 & $59 \cdot 4$ & 0.9 & $5 \cdot 0$ & $0 \cdot 4$ & $59 \cdot 2$ & 0.9 & $70 \cdot 7$ & $0 \cdot 8$ \\
\hline$\geq 30$ & $3 \cdot 6$ & 0.4 & $45 \cdot 4$ & $1 \cdot 2$ & $54 \cdot 9$ & $1 \cdot 2$ & $4 \cdot 4$ & $0 \cdot 4$ & $52 \cdot 4$ & $1 \cdot 0$ & $64 \cdot 0$ & $1 \cdot 0$ \\
\hline$P$ value for a trend & & & & & $<0 \cdot c$ & & & & & & $<0.0$ & 01 \\
\hline $\begin{array}{r}\text { Income (\$) } \\
<25000\end{array}$ & & & & & & & & & & & & \\
\hline $\begin{array}{l}<25000 \\
25000 \text { to }<50000\end{array}$ & $\begin{array}{l}3.5 \\
3 \cdot 8\end{array}$ & $\begin{array}{l}0.5 \\
0.4\end{array}$ & $\begin{array}{l}39 \cdot 3 \\
45 \cdot 6\end{array}$ & $\begin{array}{l}1 \cdot 2 \\
1 \cdot 2\end{array}$ & $\begin{array}{l}49 \cdot 0 \\
55 \cdot 8\end{array}$ & $\begin{array}{l}1 \cdot 3 \\
1 \cdot 2\end{array}$ & $\begin{array}{l}4 \cdot 1 \\
4 \cdot 8\end{array}$ & $\begin{array}{l}0.3 \\
0.4\end{array}$ & $\begin{array}{l}49 \cdot 8 \\
60 \cdot 0\end{array}$ & $\begin{array}{l}0.9 \\
0.9\end{array}$ & $\begin{array}{l}61 \cdot 0 \\
71 \cdot 0\end{array}$ & $\begin{array}{l}0.9 \\
0.8\end{array}$ \\
\hline$\geq 50000$ & $4 \cdot 1$ & 0.4 & $56 \cdot 7$ & 0.9 & $65 \cdot 8$ & 0.9 & $6 \cdot 1$ & 0.4 & $64 \cdot 7$ & $1 \cdot 0$ & $77 \cdot 9$ & 0.8 \\
\hline$P$ value for a trend & & & & & $<0 \cdot c$ & & & & & & $<0 . c$ & 001 \\
\hline Smoking & & & & & & & & & & & & \\
\hline Current smoker & $2 \cdot 9 \|$ & $0 \cdot 4$ & $41 \cdot 8 \S$ & 1.5 & $49 \cdot 9 \S$ & $1 \cdot 6$ & $4 \cdot 9$ & $0 \cdot 7$ & $50 \cdot 3 \S$ & $1 \cdot 4$ & $60 \cdot 4 \S$ & $1 \cdot 3$ \\
\hline Former smoker & $3 \cdot 9$ & $0 \cdot 4$ & $51 \cdot 7$ & 0.9 & $61 \cdot 8$ & $0 \cdot 9$ & $4 \cdot 8$ & $0 \cdot 4$ & $61 \cdot 3$ & $0 \cdot 9$ & $72 \cdot 4$ & 0.9 \\
\hline Never & $4 \cdot 3$ & $0 \cdot 4$ & $49 \cdot 8$ & $1 \cdot 1$ & $59 \cdot 7$ & $1 \cdot 1$ & $5 \cdot 3$ & $0 \cdot 3$ & $58 \cdot 9$ & $0 \cdot 7$ & $71 \cdot 7$ & 0.7 \\
\hline Heavy alcohol drinking**** & & & & & & & & & & & & \\
\hline Yes & $4 \cdot 7$ & $1 \cdot 1$ & $49 \cdot 2$ & $2 \cdot 8$ & $58 \cdot 6$ & $2 \cdot 8$ & $5 \cdot 0$ & $1 \cdot 0$ & $63 \cdot 8 \|$ & $2 \cdot 2$ & $74 \cdot 3 \|$ & $2 \cdot 0$ \\
\hline No & $3 \cdot 8$ & 0.2 & $49 \cdot 3$ & $0 \cdot 6$ & $58 \cdot 8$ & $0 \cdot 6$ & $5 \cdot 0$ & $0 \cdot 2$ & $58 \cdot 2$ & 0.5 & $70 \cdot 1$ & 0.5 \\
\hline
\end{tabular}

BRFSS, Behavioral Risk Factor Surveillance System.

${ }^{*}, t P<0.1, \ddagger P<0.01, \S P<0.001, \| P<0.05$ and for comparisons between adults with CVD and those without.

${ }_{* \star}^{*}, \ddagger P<0.01, \| P<0.05$ and for comparisons between non-Hispanic whites and other racial/ethnic groups.

${ }^{* \star *}, \S P<0.001$ and $\| P<0.05$ for comparisons between current smokers and former smokers or those who have never smoked.

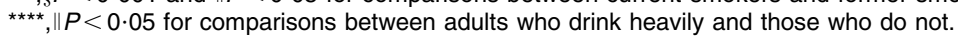

vitamin supplements as those without stroke after multivariate adjustment (Tables 3 and 4).

\section{Discussion}

Our results from a large sample showed that $58 \cdot 3 \%$ of adults aged $\geq 45$ years were taking folic acid supplements ( $4 \cdot 4 \%$ for taking less than one time per $\mathrm{d}$ and $53.9 \%$ for taking one to four times per d) and $64.6 \%$ were taking vitamin supplements regardless of their CVD status. When stratified by disease status, men with CHD were $17 \%$ more likely than those without to take folic acid supplements one to four times per d after multivariate adjustments. However, women with CHD were $44 \%$ and $32 \%$ less likely to take folic acid supplements less than one time per $\mathrm{d}$ or one to four times per $\mathrm{d}$, respectively, and $34 \%$ less likely to take vitamin supplements than those without CHD. No significant differences were observed in taking folic acid or vitamin supplements between adults with stroke and those without stroke.

The early Vitamins and Lifestyle (VITAL) study reported that adults with coronary artery disease (heart attack, bypass surgery, angioplasty or angina) were $1 \cdot 4$ times as 
Table 3 Adjusted prevalence and AOR for taking folic acid-containing vitamins among US adults aged $\geq 45$ years, by gender and by $\mathrm{CHD}$ or stroke, BRFSS, 2006

\begin{tabular}{|c|c|c|c|c|c|c|c|c|c|c|c|}
\hline \multirow{3}{*}{ Times/d } & \multicolumn{6}{|c|}{ Adjusted prevalence* } & \multicolumn{5}{|c|}{$\mathrm{AOR}^{*}$} \\
\hline & \multicolumn{2}{|c|}{$>0$ to $<1$} & \multicolumn{2}{|c|}{$1-4$} & \multicolumn{2}{|c|}{0} & \multicolumn{2}{|c|}{$>0$ to $<1$} & \multicolumn{2}{|r|}{$1-4$} & \multirow[b]{2}{*}{ Wald $P$ value } \\
\hline & $\%$ & SE & $\%$ & SE & $\%$ & SE & AOR & $95 \% \mathrm{Cl}$ & AOR & $95 \% \mathrm{Cl}$ & \\
\hline \multicolumn{12}{|c|}{ Men (n 16 772) } \\
\hline \multicolumn{12}{|c|}{ CHD } \\
\hline Yes & $3 \cdot 6+$ & $0 \cdot 6$ & $50 \cdot 4 \ddagger$ & $1 \cdot 5$ & $45 \cdot 9 \S$ & $1 \cdot 4$ & $0 \cdot 80$ & \multirow[t]{2}{*}{$0 \cdot 55,1 \cdot 19$} & $1 \cdot 17$ & \multirow[t]{2}{*}{$1 \cdot 02,1 \cdot 33$} & \multirow[t]{2}{*}{0.023} \\
\hline No & $4 \cdot 8$ & $0 \cdot 3$ & $46 \cdot 2$ & $0 \cdot 6$ & $48 \cdot 9$ & $0 \cdot 6$ & $1 \cdot 00$ & & $1 \cdot 00$ & & \\
\hline \multicolumn{12}{|l|}{ Stroke } \\
\hline Yes & $3 \cdot 9$ & $1 \cdot 4$ & $46 \cdot 8$ & $2 \cdot 8$ & $49 \cdot 3$ & $2 \cdot 8$ & $0 \cdot 82$ & \multirow[t]{2}{*}{$0 \cdot 39,1 \cdot 72$} & 0.98 & \multirow[t]{2}{*}{$0 \cdot 77,1 \cdot 24$} & \multirow[t]{2}{*}{0.865} \\
\hline No & $4 \cdot 7$ & $0 \cdot 3$ & $46 \cdot 9$ & $0 \cdot 6$ & $48 \cdot 4$ & $0 \cdot 6$ & $1 \cdot 00$ & & & & \\
\hline \multicolumn{12}{|c|}{ Women (n 25 020) } \\
\hline \multicolumn{12}{|c|}{ CHD } \\
\hline Yes & $3 \cdot 9 \S$ & $0 \cdot 6$ & $50 \cdot 0 \|$ & $1 \cdot 6$ & $46 \cdot 1 \|$ & $1 \cdot 6$ & 0.56 & \multirow[t]{2}{*}{$0 \cdot 39,0 \cdot 81$} & 0.68 & \multirow[t]{2}{*}{$0 \cdot 60,0 \cdot 79$} & \multirow[t]{2}{*}{$<0.001$} \\
\hline No & $5 \cdot 5^{\circ}$ & $0 \cdot 2$ & $57 \cdot 5$ & 0.5 & $37 \cdot 0$ & 0.5 & $1 \cdot 00$ & & $1 \cdot 00$ & & \\
\hline \multicolumn{12}{|l|}{ Stroke } \\
\hline Yes & $5 \cdot 2$ & $1 \cdot 0$ & 54.9 & $2 \cdot 2$ & $39 \cdot 8$ & $2 \cdot 2$ & 0.92 & \multirow[t]{2}{*}{$0 \cdot 60,1 \cdot 43$} & 0.91 & \multirow[t]{2}{*}{$0 \cdot 75,1 \cdot 11$} & \multirow[t]{2}{*}{0.661} \\
\hline No & $5 \cdot 4$ & $0 \cdot 2$ & $56 \cdot 8$ & 0.5 & $37 \cdot 8$ & 0.5 & $1 \cdot 00$ & & $1 \cdot 00$ & & \\
\hline
\end{tabular}

BRFSS, Behavioral Risk Factor Surveillance System; AOR, adjusted OR.

${ }^{*}$ Adjusted for age, race/ethnicity, education, BMI, income, smoking and heavy drinking.

$+P<0.1$.

$\ddagger P<0.01$.

$\S P<0.05$.

$\| P<0.001$ for comparisons between adults with CVD and those without CVD.

Table 4 Adjusted prevalence and AOR for taking any vitamins among US adults aged $\geq 45$ years, by gender and by coronary heart disease or stroke, BRFSS, 2006

\begin{tabular}{|c|c|c|c|c|c|}
\hline & \multicolumn{2}{|c|}{ Adjusted prevalence* } & \multicolumn{2}{|c|}{$\mathrm{AOR}^{*}$} & \multirow[b]{2}{*}{ Wald $P$ value } \\
\hline & $\%$ & SE & AOR & $95 \% \mathrm{Cl}$ & \\
\hline \multicolumn{6}{|c|}{ Men ( $n$ 16772) } \\
\hline \multicolumn{6}{|c|}{ CHD } \\
\hline Yes & $58 \cdot 2$ & $1 \cdot 4$ & $1 \cdot 07$ & \multirow[t]{2}{*}{$0 \cdot 94,1 \cdot 22$} & \multirow[t]{2}{*}{0.299} \\
\hline No & $56 \cdot 6$ & $0 \cdot 6$ & $1 \cdot 00$ & & \\
\hline \multicolumn{6}{|l|}{ Stroke } \\
\hline Yes & $56 \cdot 2$ & $2 \cdot 8$ & 0.97 & \multirow{2}{*}{$0 \cdot 76,1 \cdot 23$} & \multirow[t]{2}{*}{$0 \cdot 810$} \\
\hline No & $56 \cdot 9$ & $0 \cdot 6$ & $1 \cdot 00$ & & \\
\hline \multicolumn{6}{|c|}{ Women (n 25 020) } \\
\hline \multicolumn{6}{|c|}{$\mathrm{CHD}$} \\
\hline Yes & $60 \cdot 9+$ & $1 \cdot 6$ & $0 \cdot 66$ & \multirow[t]{2}{*}{$0.57,0.76$} & \multirow[t]{2}{*}{$<0.001$} \\
\hline No & $69 \cdot 9$ & 0.5 & 1.00 & & \\
\hline \multicolumn{6}{|l|}{ Stroke } \\
\hline Yes & $66 \cdot 1$ & $2 \cdot 2$ & $0 \cdot 87$ & \multirow[t]{2}{*}{$0 \cdot 71,1 \cdot 07$} & \multirow[t]{2}{*}{$0 \cdot 194$} \\
\hline No & $69 \cdot 0$ & 0.5 & $1 \cdot 00$ & & \\
\hline
\end{tabular}

BRFSS, Behavioral Risk Factor Surveillance System; AOR, adjusted OR.

${ }^{*}$ Adjusted for age, race/ethnicity, education, BMI, income, smoking and heavy drinking.

$+P<0.001$ for comparisons between adults with CVD and those without CVD.

likely to use folic acid supplements as those without coronary artery disease ${ }^{(33)}$. The data from the 1999-2002 NHANES showed that adults with either coronary artery disease or stroke were 3.6 times as likely to use folic acid supplements as those without any of the following conditions: coronary artery disease, stroke, diabetes, hypertension or hypercholesterolaemia ${ }^{(34)}$. However, in these studies, data analyses were not stratified by $\operatorname{sex}^{(33,34)}$ or by CVD status ${ }^{(34)}$. Our study demonstrates a significant gender interaction when assessing the association of taking folic acid or vitamin supplements with CHD. We found that men with CHD were significantly more likely than those without CHD to take folic acid supplements one to four times per $\mathrm{d}$ after adjustment for potential confounding variables. However, women with CHD were significantly less likely than those without CHD to take folic acid supplements or vitamin supplements. Although the role of folic acid and multivitamin interventions in primary and secondary prevention of CHD remains to be elucidated, the potential impact of not taking folic acid and vitamin supplements on the health of women with CHD needs to be studied further. Moreover, several studies have reported that folic acid supplementation significantly reduces the risk of stroke in patients with 
$\mathrm{CHD}^{(15,19,23,35)}$. Thus, our finding that men with $\mathrm{CHD}$ were significantly more likely to take folic acid supplements one to four times per $\mathrm{d}$ is of interest because this may help reduce risk of stroke in the future.

To date, studies have shown that a higher intake of folic acid from diet or supplements may help reduce risk of stroke and stroke-related mortality in primary prevention $^{(22,24,25)}$. However, its role in preventing recurrent stroke and CHD and death in patients with a history of stroke remains unclear ${ }^{(19,36)}$ and requires further investigation. Our study did not show any significant differences in the likelihood of taking folic acid or vitamin supplements between adults with stroke and those without.

In the United States, although most food is now fortified with folic acid or vitamins, dietary supplement use remains common ${ }^{(26)}$. Our results also demonstrated a higher prevalence of taking vitamin supplements and a much higher prevalence of taking folic acid supplements among participants with CHD or stroke than reported previously. In the VITAL study, $9 \cdot 2 \%$ of adults aged 50-75 years with coronary artery disease and $41.9 \%$ of adults with any CVD risk factors were taking dietary supplements; no data were reported for taking folic acid supplements among these patients ${ }^{(33)}$. In the NHANES 1999-2002, approximately $36 \%$ of adults aged $\geq 40$ years with either coronary artery disease or stroke were taking multivitamins, $8 \%$ were taking $\mathrm{B}$ vitamins, and $5 \%$ were taking folic acid as single supplements ${ }^{(34)}$. The inconsistent results of these studies may be largely due to the varying measures of folic acid or vitamin supplementation used. Nonetheless, our results are consistent with earlier studies in that the prevalence of taking folic acid or vitamin supplements was higher in women $v$. men, in former smokers $v$. current smokers, in non-Hispanic whites $v$. other racial/ethnic groups, and in those with a higher educational level or a lower $\mathrm{BMI}^{(26,33,34)}$. The question as to whether physicians or other healthcare professionals prescribed folic acid and vitamin supplements to their patients with CVD warrants further investigation.

At present, several mechanisms may help explain the possible beneficial effects of folic acid or vitamin interventions on CVD risk ${ }^{(37)}$. First, folic acid or vitamin supplementation significantly reduces carotid intima-media thickness in patients with heart disease ${ }^{(38,39)}$, a history of stroke or at risk for cerebral ischaemia ${ }^{(40,41)}$; an increased carotid intima-media thickness serves as a predictor of coronary and cerebrovascular complications ${ }^{(42)}$. Second, the antioxidant capacities of folic acid and its metabolite 5-methyltetrahydrofolate or the antioxidants from multivitamins may reduce oxidative stress and superoxide production ${ }^{(43-45)}$. Moreover, a high-dose folic acid or vitamin therapy can lower blood pressure, enhance coronary dilation in patients with coronary artery disease ${ }^{(46)}$ and improve endothelial function in patients with metabolic syndrome, CHD or diabetes ${ }^{(43,45,47-49)}$; these improvements are independent of homocysteine lowering. In addition, the use of multivitamins reduces C-reactive protein levels, a risk factor for $\mathrm{CVD}^{(50)}$.

\section{Study limitations and strengths}

Our study was subjected to several limitations. First, all information including folic acid or vitamin supplementation and CVD status was self-reported, and thus subject to recall bias. Second, although we have assessed the frequency of folic acid supplementation, the levels of folic acid and vitamin intake were unknown in the present study. In addition, data on the biomarkers of folic acid and vitamin supplementation were also unknown. Moreover, data on folic acid intake from fortified grain products were not collected in the study and therefore we were unable to adjust for this factor in our analyses. Finally, the use of medications for coronary artery disease or stroke has been shown to affect the behaviours of taking folic acid or vitamin supplements among these patients ${ }^{(34)}$; however, in the present study, information on medications for CHD or stroke was not collected and therefore we were unable to conduct stratified analyses by medications. Despite these limitations, the results of the present study were strengthened by the large sample size from a population-based survey, and the analyses were stratified by sex and by diseases.

\section{Conclusions}

Among adults aged $\geq 45$ years, substantial variations exist in the prevalence and likelihood of taking folic acid or vitamin supplements by gender and by CHD status, but not by stroke status. Men with CHD had a significantly increased likelihood of taking folic acid supplements; whereas women with CHD had a significantly decreased likelihood of take folic acid or vitamin supplements. Our results provide fundamental knowledge for clinical physicians, nutritionists and dietitians on the behaviours of taking folic acid and vitamin supplementation among patients with CHD or stroke.

\section{Acknowledgements}

The authors thank the BRFSS coordinators for their work on the survey. Disclaimer: The findings and conclusions in this article are those of the authors and do not necessarily represent the official position of the Centers for Disease Control and Prevention. Sources of funding: This research received no specific grant from any funding agency. Authors' contributions: Each author has contributed significantly to this work. G.Z. obtained the data from BRFSS, conducted the data analyses, interpreted the data and prepared the paper. E.S.F. supervised the data 
analyses and contributed to the writing of the manuscript. E.S.F, C.L. and A.H.M. revised the paper for important intellectual content. All authors critically reviewed and approved the final version of the paper. Conflict of interest declaration: The authors have no financial support or potential conflicts of interest to disclose.

\section{References}

1. Ford ES, Ajani UA, Croft JB et al. (2007) Explaining the decrease in US deaths from coronary disease, 1980-2000. $N$ Engl J Med 356, 2388-2398.

2. Rosamond W, Flegal K, Furie K et al. (2008) Heart disease and stroke statistics - 2008 update: a report from the American Heart Association Statistics Committee and Stroke Statistics Subcommittee. Circulation 117, e25-e146.

3. Clarke R, Collins R, Lewington S et al. (2002) The homocysteine studies collaboration, homocysteine and risk of ischemic heart disease and stroke: a meta-analysis. JAMA 288, 2015-2022.

4. Ford ES, Smith SJ, Stroup DF et al. (2002) Homocyst(e)ine and cardiovascular disease: a systematic review of the evidence with special emphasis on case-control studies and nested case-control studies. Int J Epidemiol 31, 59-70.

5. Wald NJ, Watt HC, Law MR et al. (1998) Homocysteine and ischaemic heart disease: results of a prospective study with implications regarding prevention. Arch Intern Med 158, 862-867.

6. Clarke R, Frost C, Sherliker P et al. (2005) Homocysteine Lowering Trialists' Collaboration. Dose-dependent effects of folic acid on blood concentrations of homocysteine: a meta-analysis of the randomised trials. Am J Clin Nutr 82, 806-812.

7. Neal B, MacMahon S, Ohkubo $T$ et al. (2002) Dosedependent effects of folic acid on plasma homocysteine in a randomised trial conducted among 723 individuals with coronary heart disease. Eur Heart J 23, 1509-1515.

8. Holmquist C, Larsson S, Wolk A et al. (2003) Multivitamin supplements are inversely associated with risk of myocardial infarction in men and women - Stockholm Heart Epidemiology Program (SHEEP). J Nutr 133, 2650-2654.

9. Ishihara J, Iso $\mathrm{H}$, Inoue $\mathrm{M}$ et al. (2008) Intake of folate, vitamin B6 and vitamin B12 and the risk of CHD: the Japan Public Health Centre-based Prospective Study Cohort I. J Am Coll Nutr 27, 127-136.

10. Rimm EB, Willett WC, Hu FB et al. (1998) Folate and vitamin B6 from diet and supplements in relation to risk of coronary heart disease among women. JAMA 279, 359-364.

11. Tavani A, Pelucchi C, Parpinel M et al. (2004) Folate and vitamin $\mathrm{B}(6)$ intake and risk of acute myocardial infarction in Italy. Eur J Clin Nutr 58, 1266-1272.

12. Schnyder G, Roffi M, Pin R et al. (2001) Decreased rate of coronary restenosis after lowering of plasma homocysteine levels. $N$ Engl J Med 345, 1593-1600.

13. Schnyder G, Roffi M, Flammer Y et al. (2002) Effect of homocysteine-lowering therapy with folic acid, vitamin B12, and vitamin B6 on clinical outcome after percutaneous coronary intervention: the Swiss Heart study: a randomised controlled trial. JAMA 288, 973-979.

14. Bazzano LA, Reynolds K, Holder KN et al. (2006) Effect of folic acid supplementation on risk of cardiovascular diseases: a meta-analysis of randomised controlled trials. JAMA 296, 2720-2726.

15. Bønaa KH, Njølstad I, Ueland PM et al. (2006) Homocysteine lowering and cardiovascular events after acute myocardial infarction. N Engl J Med 354, 1578-1588.
16. Ebbing M, Bleie $\varnothing$, Ueland PM et al. (2008) Mortality and cardiovascular events in patients treated with homocysteine-lowering $\mathrm{B}$ vitamins after coronary angiography: a randomised controlled trial. JAMA 300, 795-804.

17. Muntwyler J, Hennekens CH, Manson JE et al. (2002) Vitamin supplement use in a low-risk population of US male physicians and subsequent cardiovascular mortality. Arch Intern Med 162, 1472-1476.

18. Albert CM, Cook NR, Gaziano JM et al. (2008) Effect of folic acid and B vitamins on risk of cardiovascular events and total mortality among women at high risk for cardiovascular disease: a randomised trial. JAMA 299, 2027-2036.

19. Lonn E, Yusuf S, Arnold MJ et al. (2006) Homocysteine lowering with folic acid and B vitamins in vascular disease. N Engl J Med 354, 1567-1577.

20. Yang Q, Botto LD, Erickson JD et al. (2006) Improvement in stroke mortality in Canada and the United States, 1990 to 2002. Circulation 113, 1335-1343.

21. Food and Drug Administration (1996) Food standards: amendment of standards of indentity for enriched cereal grain products to require addition of folic acid. Fed Regist 61, 8781-9797.

22. Bazzano LA, He J, Ogden LG et al. (2002) Dietary intake of folate and risk of stroke in US men and women: NHANES I Epidemiologic Follow-up Study. National Health and Nutrition Examination Survey. Stroke 33, 1183-1188.

23. Sepe V, Adamo G, Giuliano MG et al. (2007) Folic acid for stroke prevention - author reply 651. Lancet 370, 651.

24. Virtanen JK, Voutilainen S, Happonen P et al. (2005) Serum homocysteine, folate and risk of stroke: Kuopio Ischaemic Heart Disease Risk Factor (KIHD) Study. Eur J Cardiovasc Prev Rehabil 12, 369-375.

25. Wang X, Qin X, Demirtas H et al. (2007) Efficacy of folic acid supplementation in stroke prevention: a metaanalysis. Lancet 369, 1876-1882.

26. Radimer K, Bindewald B, Hughes J et al. (2004) Dietary supplement use by US adults: data from the National Health and Nutrition Examination Survey, 1999-2000. Am J Epidemiol 160, 339-349.

27. Mokdad AH, Stroup DF \& Giles WH (2003) Public health surveillance for behavioural risk factors in a changing environment. Recommendations from the Behavioural Risk Factor Surveillance Team. MMWR Recomm Rep 52, 1-12.

28. Nelson DE, Holtzman D, Bolen J et al. (2001) Reliability and validity of measures from the Behavioural Risk Factor Surveillance System (BRFSS). Soc Prev Med 46, Suppl. 1, S3-S42.

29. Nelson DE, Powell-Griner E, Town M et al. (2003) A comparison of national estimates from the National Health Interview Survey and the Behavioural Risk Factor Surveillance System. Am J Public Health 93, 1335-1341.

30. Satia-Abouta J, Patterson RE, King IB et al. (2003) Reliability and validity of self-report of vitamin and mineral supplement use in the vitamins and lifestyle study. Am J Epidemiol 157, 944-954.

31. Bush TL, Miller SR, Golden AL et al. (1989) Self-report and medical records report agreement of selected medical conditions in the elderly. Am J Public Health 79, 1554-1556.

32. Okura Y, Urban LH, Mahoney DW et al. (2004) Agreement between self-report questionnaires and medical record data was substantial for diabetes, hypertension, myocardial infarction and stroke but not for heart failure. $J$ Clin Epidemiol 57, 1096-1103.

33. Satia-Abouta J, Kristal AR, Patterson RE et al. (2003) Dietary supplement use and medical conditions: the VITAL study. Am J Prev Med 24, 43-51.

34. Buettner C, Phillips RS, Davis RB et al. (2007) Use of dietary supplements among United States adults with coronary 
artery disease and atherosclerotic risks. $A m$ J Cardiol 99, 661-666.

35. Liem A, Reynierse-Buitenwerf GH, Zwinderman AH et al (2005) Secondary prevention with folic acid: results of the Goes extension study. Heart 91, 1213-1214.

36. Toole JF, Malinow MR, Chambless LE et al. (2004) Lowering homocysteine in patients with ischaemic stroke to prevent recurrent stroke, myocardial infarction, and death: the Vitamin Intervention for Stroke Prevention (VISP) randomised controlled trial. JAMA 291, $565-575$.

37. Moens AL, Vrints CJ, Claeys MJ et al. (2008) Mechanisms and potential therapeutic targets for folic acid in cardiovascular disease. Am J Physiol Heart Circ Physiol 294, H1971-H1977.

38. Fernandez-Miranda C, Yebra M, Aranda JL et al. (2007) Effect of folic acid treatment on carotid intima-media thickness of patients with coronary disease. Int J Cardiol 118, 345-349.

39. Ntaios G, Savopoulos C, Hatzitolios A et al. (2009) Is there a beneficial effect of folic acid on carotid intima-media thickness? Int J Cardiol 135, 260-261.

40. Potter K, Hankey GJ, Green DJ et al. (2008) The effect of long-term homocysteine-lowering on carotid intimamedia thickness and flow-mediated vasodilation in stroke patients: a randomised controlled trial and meta-analysis. BMC Cardiovasc Disord 8, 24-37.

41. Till U, Rohl P, Jentsch A et al. (2005) Decrease of carotid intima-media thickness in patients at risk to cerebral ischaemia after supplementation with folic acid, vitamins B6 and B12. Atherosclerosis 181, 131-135.

42. Poredos P (2004) Intima-media thickness: indicator of cardiovascular risk and measure of the extent of atherosclerosis. Vasc Med 9, 46-54.
43. Antoniades C, Shirodaria C, Warrick N et al. (2006) 5-Methyltetrahydrofolate rapidly improves endothelial function and decreases superoxide production in human vessels: effects on vascular tetrahydrobiopterin availability and endothelial nitric oxide synthase coupling. Circulation 114, 1193-1201.

44. Nakano E, Higgins JA \& Powers HJ (2001) Folate protects against oxidative modification of human LDL. Br J Nutr 86, 637-639.

45. Shirodaria C, Antoniades C, Lee J et al. (2007) Global improvement of vascular function and redox state with low-dose folic acid: implications for folate therapy in patients with coronary artery disease. Circulation 115, 2262-2270.

46. Tawakol A, Migrino RQ, Aziz KS et al. (2005) High-dose folic acid acutely improves coronary vasodilator function in patients with coronary artery disease. J Am Coll Cardiol 45, 1580-1584.

47. Doshi SN, McDowell IF, Moat SJ et al. (2002) Folic acid improves endothelial function in coronary artery disease via mechanisms largely independent of homocysteine lowering. Circulation 105, 22-26.

48. Setola E, Monti LD, Galluccio E et al. (2004) Insulin resistance and endothelial function are improved after folate and vitamin B12 therapy in patients with metabolic syndrome: relationship between homocysteine levels and hyperinsulinemia. Eur J Endocrinol 151, 483-489.

49. Title LM, Ur E, Giddens K et al. (2006) Folic acid improves endothelial dysfunction in type 2 diabetes - an effect independent of homocysteine-lowering. Vasc Med 11, 101-109.

50. Church TS, Earnest CP, Wood KA et al. (2003) Reduction of $\mathrm{C}$-reactive protein levels through use of a multivitamin. Am J Med 115, 702-707. 